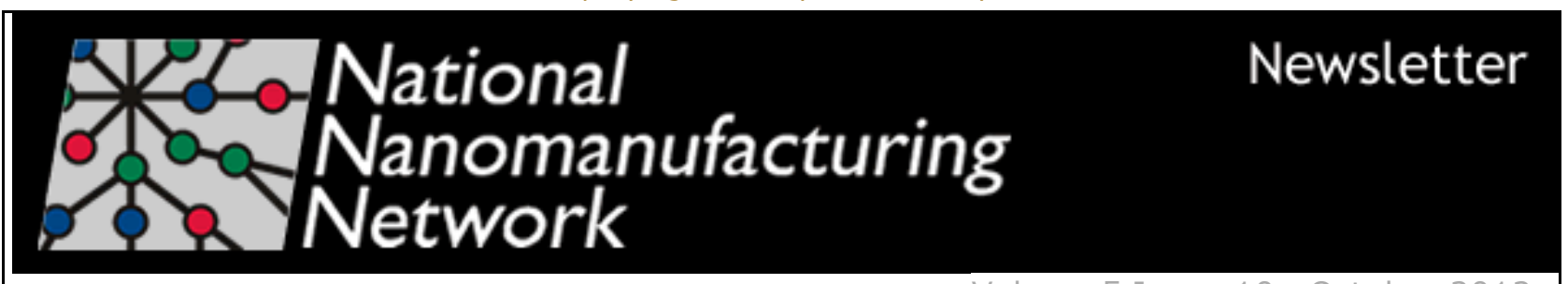

Volume 5 Issue 10 - October 2012

\section{Nanomanufacturing \\ Innovations Impact Future Industry Roadmap Directions}

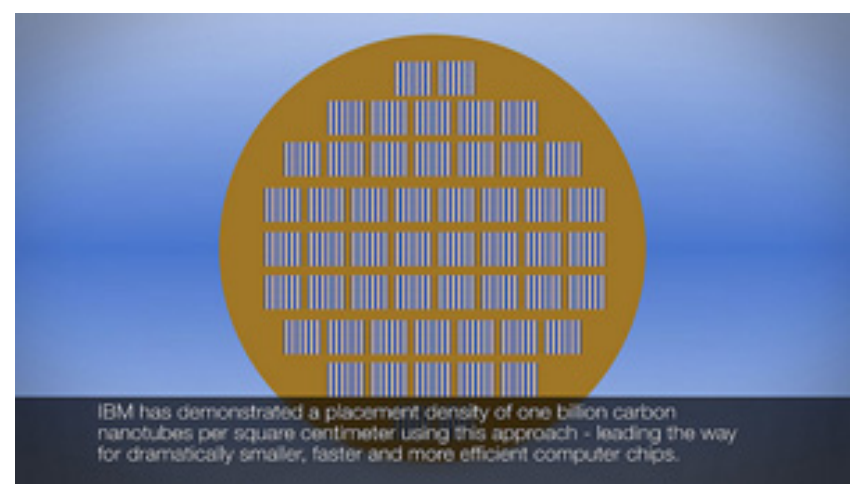

Nanomanufacturing innovations have made numerous impacts in various industry roadmaps with semiconductor, data storage, and aerospace being prime examples. For many of these examples, the impact of nanotechnology provides a marginal extension of the industry roadmap, as in the case of directed self-assembly (DSA) for bit patterned media magnetic data storage, and extending the utility of specific lithographic tools. In the case of new nanotechnology-enabled composite materials, the aerospace industry has already reaped significant benefit, and advances in the value chain will impact everything from sporting equipment to automobiles, thereby providing the promise of significant economic and societal benefit that typically follows such discussions on nanotechnology. More....

Regards,

Jeff Morse, Managing Director,

National Nanomanufacturing Network

Learn More about the

NanoBusiness Interview with Paul Clayson, President \& CEO, HzO, Inc.

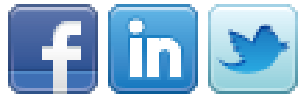

Advertisements

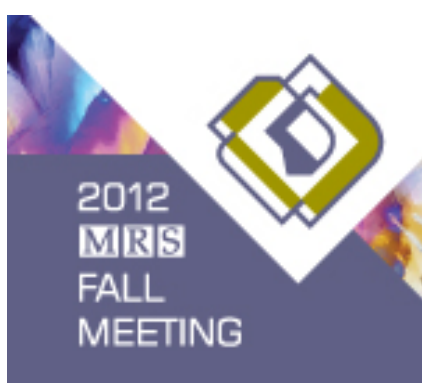

\section{Job Opportunities}

R\&D Scientist

SouthWest NanoTechnologies, Inc.

Assistant/Associate/Full

Professor - College of

Engineering

Northeastern University

Assistant/Associate/Full

Professor - Advanced

Manufacturing

University of Texas at Austin

\section{Upcoming Events}

November 4-6, 2012

Sustainable Nanotechnology

Organization Conference

November 13-15, 2012

Nanosafe 2012

November 26-30, 2012

MRS Fall Meeting 2012

View Full Calendar 


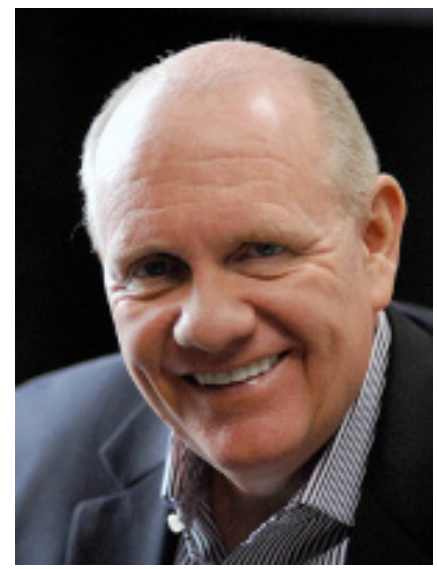

In this month's

interview, we talk with

Paul Clayson, President

\& $\mathrm{CEO}$ of $\mathrm{HzO}$, Inc. We

were delighted to have

Mr. Clayson speak at our

11th Annual

NanoBusiness

Conference in Boston, MA and the Nanotech

Commercialization

Conference in Durham,

NC. $\mathrm{HzO}$ is a Salt Lake City, Utah based start-up with cutting-edge, breakthrough technology that makes electronic devices water resistant. Mr. Clayson has been a business owner, global strategic planning expert, financial and investment strategist and senior political advisor for the past 30 years. Mr. Clayson previously served as Chairman and Chief Executive Officer of nCoat, Inc., an award winning nanotechnology materials development and manufacturing company, which he sold in 2010. He has served as President and COO of Sequoia Pacific Research Company. More...

\section{Nanotextured Surfaces Impregnated with Lubricant for Enhanced Condensation}

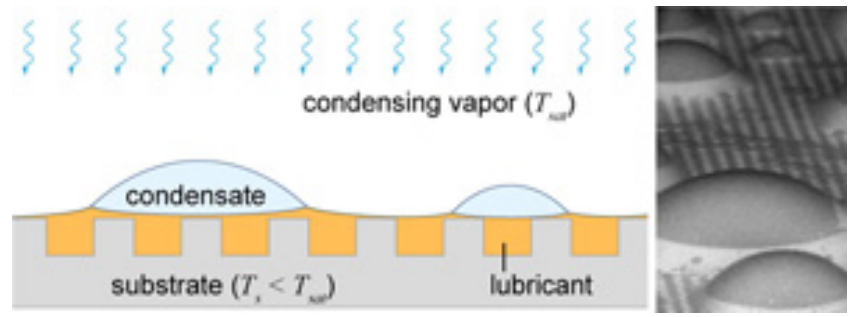

Incorporation of specific nanostructures and textured patterns on surfaces is a growing area of research for controlling surface wetting properties. By tailoring the hydrophobicity for a given surface, enhanced features may be achieved by design such as anti-icing or anti-fog, self-cleaning and repellency to a range of liquids. Another significant area of application for such surfaces is condensation for industrial processes such as power generation, thermal management, air conditioning, desalination, and transportation. Enhancing the heat transfer during condensation will significantly impact the energy efficiency for these applications, further reducing cost and
Featured Video

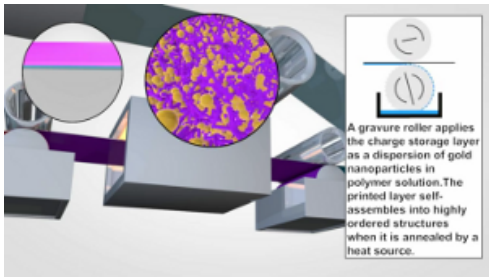

Roll-to-Roll Fabrication of a Floating Gate Transistor.

Advertisements

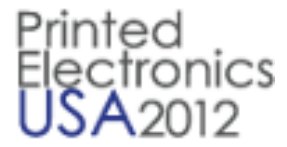

December 5-6, 2012 Santa Clara, CA, USA

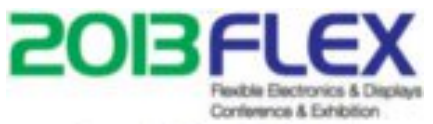

Jan 29 - Feb 1 Phoenix, Arizona Flextech, suear

\section{Recently Published From Our Affiliates}

A comparison of homogenous and separated flow assumptions for adiabatic capillary flow Applied Thermal Engineering 48: 186-193

Multi-scale alignment and positioning system - MAPS Precision Engineering Journal of the International Societies for Precision Engineering and Nanotechnology 36(4): 517$\underline{537}$

Understanding and correcting for carbon nanotube interferences with a commercial LDH cytotoxicity assay Toxicology 299(2-3): 99-111

The interplay of monolayer structure and serum protein interactions on the cellular uptake of gold nanoparticles 
physical impact on the system. Depending on the wettability of the surface, the condensate either forms as a film or as discrete drops on the surface. The latter is more desirable for effective heat transfer, as a film can behave as a thermal barrier, while the dropwise condensate can be continuously shed allowing for renewed nucleation and growth of drops. As a result of these possible advantages, there has been significant interest in developing superhydrophobic surfaces to promote dropwise condensation. Such surfaces to date have typically incorporated complex surface patterns to obtain extreme non-wetting properties in which droplets are formed and sit atop the surface features. In many cases, the droplets suffer from poor surface mobility and become pinned to the surface features. A possible solution to this issue is intricate surface textures that promote shedding of the coalesced droplets, but these are difficult to create and susceptible to damage.

More....

\section{Made in IBM Labs: Researchers Demonstrate Initial Steps toward Commercial Fabrication of Carbon Nanotubes as a Successor to Silicon}

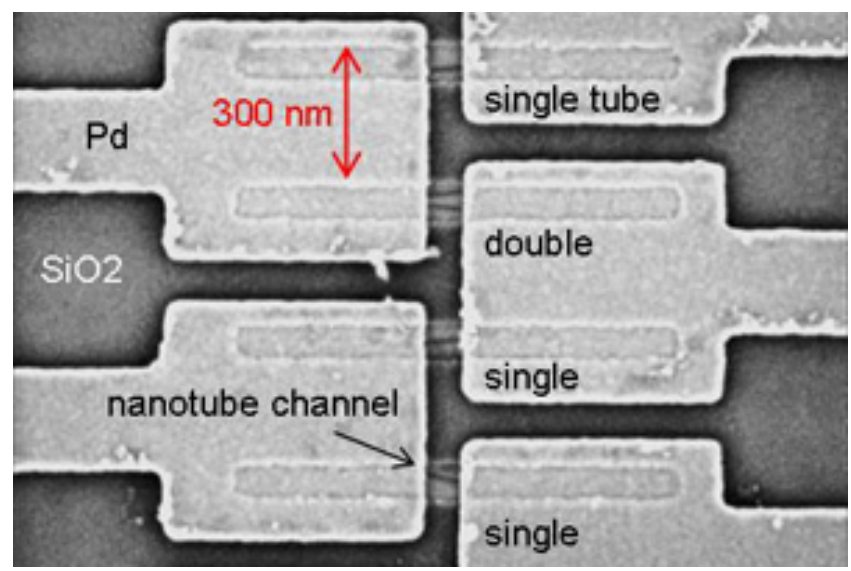

- For the first time, scientists precisely place and test more than ten thousand carbon nanotube devices in a single chip using mainstream manufacturing processes

- Novel processing method helps pave the way for carbon technology as a viable alternative to silicon in future computing

IBM (NYSE: IBM) scientists have demonstrated a new approach to carbon nanotechnology that opens up the path for commercial fabrication of
Small 8(17): 2659-2663

Understanding anomalous current-voltage characteristics in microchannel-nanochannel interconnect devices Journal of Colloid and Interface Science 384: 162-171

\section{Affiliated Centers}
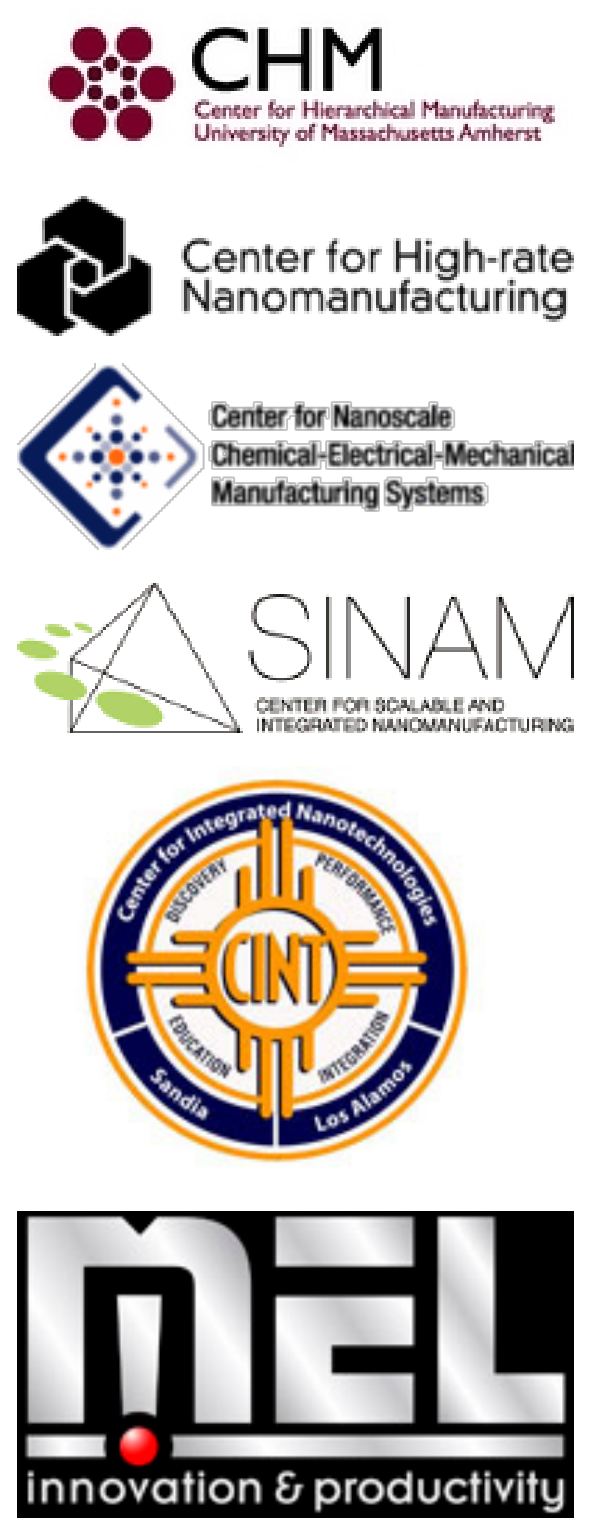
dramatically smaller, faster and more powerful computer chips. For the first time, more than ten thousand working transistors made of nano-sized tubes of carbon have been precisely placed and tested in a single chip using standard semiconductor processes. These carbon devices are poised to replace and outperform silicon technology allowing further miniaturization of computing components and leading the way for future microelectronics.

More....

\section{Read more on InterNano}

Subscribe / Unsubscribe from this list.

Our mailing address is:

The National Nanomanufacturing Network

374 Lederle Graduate Research Center

710 N. Pleasant Street

University of Massachusetts

Amherst, MA 01003

Our email address is:

nnn@nanomanufacturing.org

Our phone number is:

(413) 577-0570

Copyright (C) 2012 The National Nanomanufacturing Network All rights reserved.

Supported by the National Science Foundation under Grant No. CMMI-1025020. 